\title{
Multi-Channel 40 Gbit/s NRZ-DPSK Demodulation Using a Single Silicon Microring Resonator
}

Ding, Yunhong; Xu, Jing; Peucheret, Christophe; Pu, Minhao; Liu, Liu; Seoane, Jorge; Ou, Haiyan; Zhang, Xinliang; Huang, Dexiu

Published in:

Journal of Lightwave Technology

Link to article, DOI:

10.1109/JLT.2010.2101049

Publication date:

2011

Document Version

Publisher's PDF, also known as Version of record

Link back to DTU Orbit

Citation $(A P A)$ :

Ding, Y., Xu, J., Peucheret, C., Pu, M., Liu, L., Seoane, J., Ou, H., Zhang, X., \& Huang, D. (2011). Multi-Channel 40 Gbit/s NRZ-DPSK Demodulation Using a Single Silicon Microring Resonator. Journal of Lightwave Technology, 29(5), 677-684. https://doi.org/10.1109/JLT.2010.2101049

\section{General rights}

Copyright and moral rights for the publications made accessible in the public portal are retained by the authors and/or other copyright owners and it is a condition of accessing publications that users recognise and abide by the legal requirements associated with these rights.

- Users may download and print one copy of any publication from the public portal for the purpose of private study or research.

- You may not further distribute the material or use it for any profit-making activity or commercial gain

- You may freely distribute the URL identifying the publication in the public portal 


\title{
Multi-Channel 40 Gbit/s NRZ-DPSK Demodulation Using a Single Silicon Microring Resonator
}

\author{
Yunhong Ding, Jing Xu, Christophe Peucheret, Minhao Pu, Liu Liu, Jorge Seoane, Haiyan Ou, \\ Xinliang Zhang, and Dexiu Huang
}

\begin{abstract}
We comprehensively analyze the demodulation of wavelength division multiplexed (WDM) non return-to-zero differential phase-shift keying (NRZ-DPSK) signals by a single microring resonator. Simultaneous demodulation of multiple $\mathbf{4 0}$ Gbit/s WDM NRZ-DPSK channels is demonstrated using a single silicon microring resonator with free spectral range (FSR) of 100 GHz. Bit error measurements show very good performance for both through and drop port demodulations for all channels, and the drop port demodulation exhibits better wavelength detuning tolerance than for demodulation using a Mach-Zehnder delay interferometer (MZDI).
\end{abstract}

Index Terms-Demodulation, differential phase shift keying (DPSK), microring resonator, silicon photonics.

\section{INTRODUCTION}

D IFFERENTIAL phase-shift keying (DPSK) is a promising modulation format for optical communication networks. Compared to on-off keying (OOK) formats, DPSK exhibits a $3 \mathrm{~dB}$ improvement in receiver sensitivity when balanced detection is employed, and is more tolerant to fiber nonlinearities [1]. Delay interferometers (DI), in which one-bit delay between the two arms of the interferometer realizes conversion from phase modulation to intensity modulation, are typically applied to demodulate DPSK signals and have been implemented as Mach-Zehnder [2] or Michelson structures [3]. The use of delay interferometers relying on a birefringent fiber has also been reported [4], [5]. Other approaches have also been proposed and demonstrated, including the use of optical bandpass filters [6] or of a delay-asymmetric nonlinear loop mirror [7]. One important issue to be taken into account in

Manuscript received June 29, 2010; revised October 29, 2010; accepted December 07, 2010. Date of publication December 20, 2010; date of current version February 25, 2011. This work was supported in part by the Villum Kann Rasmussen Foundation through the Nanophotonics for Terabit Communications (NATEC) Centre of Excellence and by the National Natural Science Foundation of China under Grant 60867002.

Y. Ding is with the Wuhan National Laboratory for Optoelectronics, School of Optoelectronics Science and Engineering, Huazhong University of Science and Technology, Wuhan, 430074 Hubei, China, and also with the Department of Photonics Engineering, Technical University of Denmark, 2800 Kgs. Lyngby, Denmark.

J. Xu, C. Peucheret, M. Pu, L. Liu, J. Seoane, and H. Ou are with the Department of Photonics Engineering, Technical University of Denmark, $2800 \mathrm{Kgs}$. Lyngby, Denmark (e-mail: haou@fotonik.dtu.dk).

X. Zhang and D. Huang are with the Wuhan National Laboratory for Optoelectronics, School of Optoelectronics Science and Engineering, Huazhong University of Science and Technology, Wuhan, 430074 Hubei, China (e-mail: wnlo2@mail.hust.edu.cn).

Color versions of one or more of the figures in this paper are available online at http://ieeexplore.ieee.org.

Digital Object Identifier 10.1109/JLT.2010.2101049 photonic integrated circuits is the possible polarization dependence of the demodulator, which can be reduced by appropriate techniques [8].

In wavelength division multiplexing (WDM) networks, the use of a single device to simultaneously demodulate a number of channels would offer a much preferred cost effective solution. Such an approach has been explored using frequency periodic filters, including fibre Bragg gratings arrays [9], sampled fiber gratings [10], delay interferometers [11] and arrayed waveguide grating (AWG) de-multiplexers [12].

Silicon microring resonators (MRRs) are compact and versatile devices whose use as DPSK demodulators has been recently proposed [13] and demonstrated [14] for single channel operation. This scheme is also foreseen to be very attractive for multi-channel applications due to its compact size, integration ability, and CMOS compatible fabrication process. However the optimization of MRRs for WDM demodulation of DPSK signals has not been reported so far.

In this paper, we comprehensively analyze the performance of WDM demodulation of non return-to-zero DPSK (NRZ-DPSK) signals at $40 \mathrm{Gbit} / \mathrm{s}$ based on a single MRR. Multiple channels WDM NRZ-DPSK demodulation is demonstrated at $40 \mathrm{Gbit} / \mathrm{s}$ by a single silicon MRR with free spectral range (FSR) of $100 \mathrm{GHz}$ and $\mathrm{Q}$ value of 6700 . Both the through and drop port demodulations exhibit very good and similar bit error ratio (BER) performance for all the channels. The wavelength detuning tolerance is further experimentally characterized, showing similar wavelength detuning sensitivity compared to Mach-Zehnder delay interferometers (MZDIs) for through port demodulation, and improved wavelength detuning tolerance for drop port demodulation.

\section{PRINCIPLE OF OPERATION AND SimUlations}

\section{A. Principle}

The principle of NRZ-DPSK demodulation using a MRR is to convert phase modulation to amplitude-modulation [12] by exploiting the discriminating filter property of the MRR transfer function. The general layout of the add/drop MRR used in the present work is represented in Fig. 1.

The transfer functions of the through and drop ports of the MRR can be expressed as

$$
\begin{aligned}
t_{\text {through }} & =\frac{r[1-a \exp (-j \theta)]}{1-a r^{2} \exp (-j \theta)} \\
t_{\text {drop }} & =-\frac{\kappa^{2} \sqrt{a} \exp (-j \theta / 2)}{1-a r^{2} \exp (-j \theta)}
\end{aligned}
$$




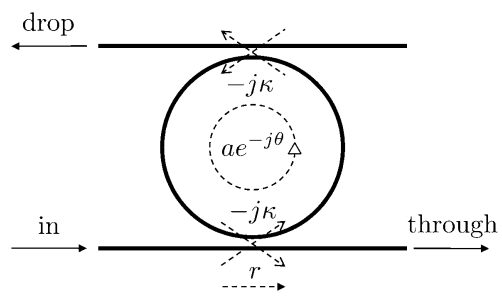

Fig. 1. General layout of an add/drop MRR.

where it is assumed that the MRR is geometrically symmetrical with identical through and drop coupling regions. $r$ and $\kappa$ are the field transmission and coupling coefficients of the coupling regions of the resonator, respectively, satisfying the relation $|r|^{2}+|\kappa|^{2}=1$ for lossless coupling. $\theta$ and $a$ are the roundtrip phase shift and field transmission coefficients along the ring waveguide, respectively. NRZ-DPSK signals can be demodulated by the MRR, resulting in the generation of alternate-mark inversion (AMI) and duobinary (DB) signals [13] at the through and drop ports, respectively. Thanks to the periodic nature of the frequency response of the MRR, simultaneous multiple WDM NRZ-DPSK demodulation can be achieved by tuning the carrier wavelength of each channel so that it coincides with one of the notches of the through transfer function. The demodulated AMI signals can then be wavelength de-multiplexed using an optical bandpass filter (OBPF) or a wavelength de-multiplexer such as an AWG. Similar multi-channel operation can also be achieved with the drop port of the MRR, resulting in the generation of DB signals.

\section{B. Influences of $Q$ Value of MRR and Bandwidth of $O B P F$}

For MRR-based WDM NRZ-DPSK demodulation, the demodulation performance is influenced not only by the $\mathrm{Q}$ value of the MRR, but also by the bandwidth of the OBPF or AWG used for de-multiplexing. In this section, the influence of the MRR and OBPF bandwidth on the performance of NRZ-DPSK demodulation is analyzed numerically for $40 \mathrm{Gbit} / \mathrm{s}$ operation. In the following analysis, the transfer function of the de-multiplexing OBPF is modeled as a 1st order Gaussian type filter and its bandwidth is defined as full-width at half-maximum (FWHM). The Karhunen-Loève expansion method [15], [16] is applied to evaluate the BER performance for a receiver, which is assumed to be limited by amplified spontaneous emission (ASE) noise. Pre and post-detection filters modeled as 1st order Gaussian response with FWHM bandwidth of $320 \mathrm{GHz}$ and 5th order Bessel response with 3-dB cut-off frequency of $30 \mathrm{GHz}$ are employed in the numerical analysis. Throughout this paper, the optical signal-to-noise ratio (OSNR) is defined with a $0.1 \mathrm{~nm}$ bandwidth for the integration of the ASE noise spectral density. A pseudo-random binary sequence (PRBS) of length $2^{7}-1$ is adopted for all simulations. Fig. 2(a) and (b) shows the required OSNR (to obtain a BER of $10^{-9}$ ) for the MRR demodulated AMI and DB signals under different bandwidths of the de-multiplexing OBPF and power coupling coefficients $|\kappa|^{2}$ (or equivalently $\mathrm{Q}$ values) of the MRR. One can find that, as the bandwidth of the OBPF increases, and $|\kappa|^{2}$ increases (or the corresponding Q value of the MRR decreases), a smaller OSNR is required to obtain a BER of $10^{-9}$ for the AMI signal
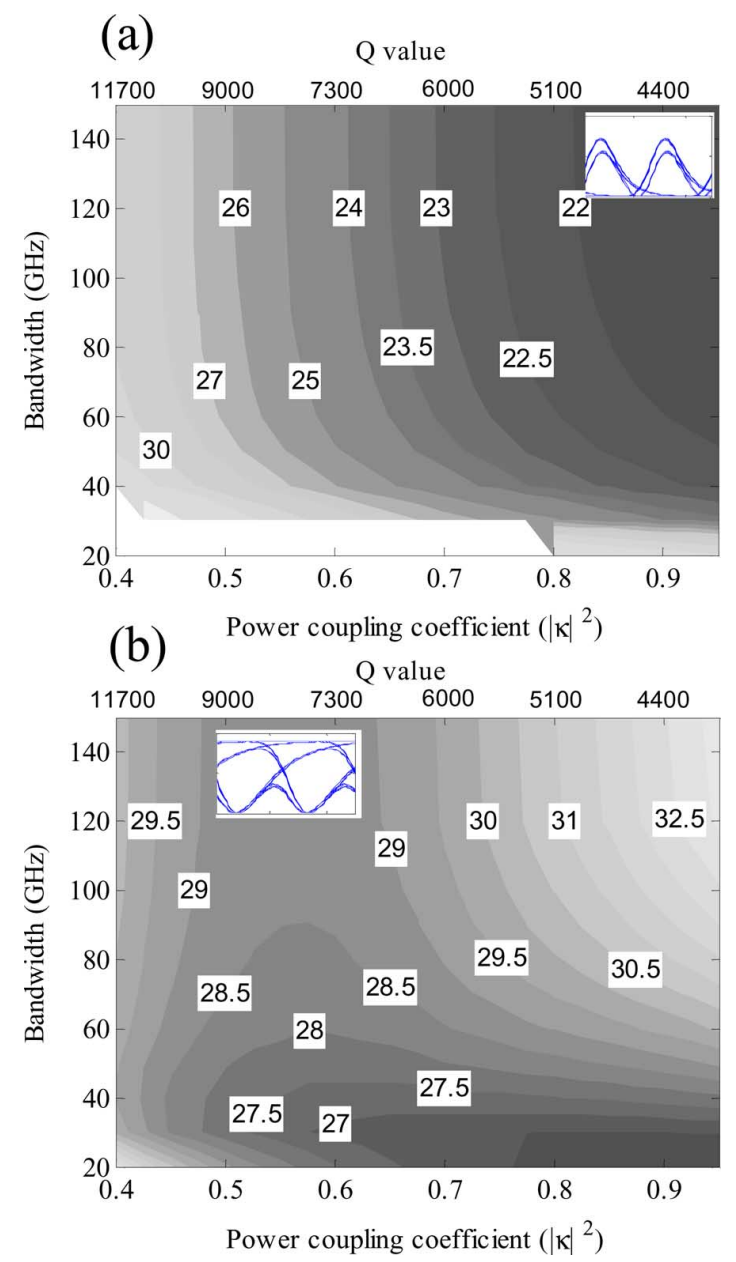

Fig. 2. Simulated required OSNR (to obtain a BER of $10^{-9}$ ) at a BER of $40 \mathrm{Gbit} / \mathrm{s}$ versus power coupling coefficient of the MRR and $3 \mathrm{~dB}$ bandwidth of the de-multiplexing OBPF for (a) through and (b) drop ports of the MRR. $a=0.96$. The insets represent the simulated eye-diagram with de-multiplexing OBPF bandwidth of $65 \mathrm{GHz}$ and MRR coupling coefficient $|\kappa|^{2}=0.65$.

at the through port, as shown in Fig. 2(a). In this case, the notch in the transfer function of the MRR mostly determines the phase-to-intensity conversion performance and the OBPF bandwidth has little influence, provided it is sufficiently large not to truncate the demodulated signal spectrum. However, for the demodulated DB signal at the drop port, an optimum OBPF bandwidth should be chosen depending on the $|\kappa|^{2}$ value in order to achieve the lowest OSNR requirement, as shown in Fig. 2(b). This is because the demodulation at the drop port essentially depends on the OBPF bandwidth, since in this case the transfer function of the MRR is nearly constant over most of the NRZ-DPSK signal bandwidth for low Q values of the MRR. The dependence of the demodulation performance at the drop port on the OBPF bandwidth is in agreement with earlier results where a single OBPF was used for demodulation and where an optimum bandwidth was also identified [6].

Fig. 3 shows a comparison of the OSNR requirement to achieve a BER of $10^{-9}$ as a function of the OBPF FWHM bandwidth when demodulation is performed using either a MZDI followed by an OBPF, a MRR followed by an OBPF, or only an OBPF. One can find that, as the bandwidth of the OBPF decreases, the AMI and DB signals demodulated by the MZDI 


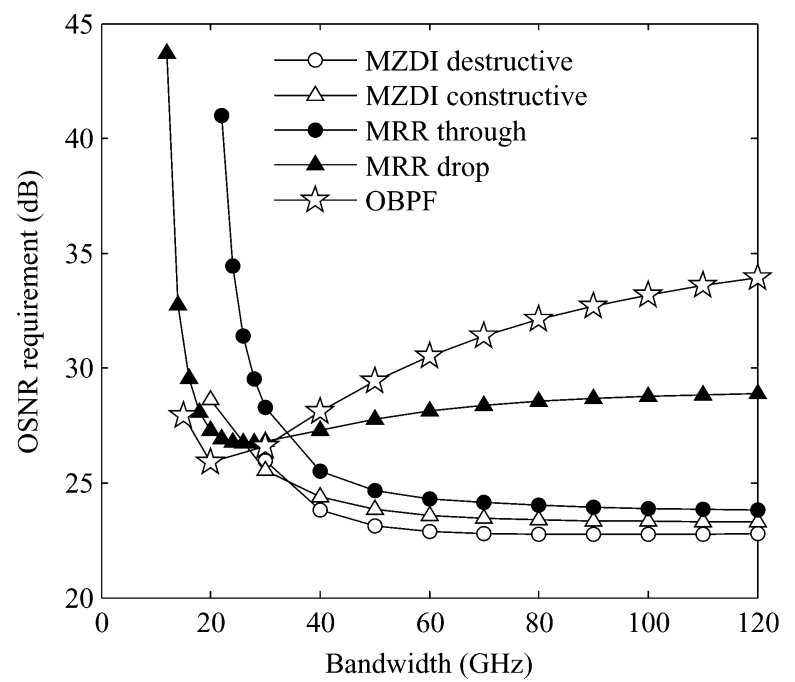

Fig. 3. Simulated required OSNR (to obtain a BER of $10^{-9}$ ) as a function of the bandwidth of the OBPF for the demodulation of $40 \mathrm{Gbit} / \mathrm{s}$ NRZ-DPSK signals using a MZDI followed by an OBPF, a MRR followed by an OBPF as well as only an OBPF. All devices are assumed to be perfectly tuned to the NRZ-DPSK signal centre frequency. $a=0.96$ and $|\kappa|^{2}=0.63$ for the MRR.

and the DB signal demodulated by the MRR are consistently degraded. However, an optimum bandwidth exists for the DB signal demodulated at the drop port of the MRR and the signal demodulated by an OBPF alone. This optimum bandwidth is equal to about $30 \mathrm{GHz}$ for MRR demodulation, while it is found to be equal to about 0.6 times the bit rate, corresponding to $24 \mathrm{GHz}$, for OBPF demodulation, in agreement with [6]. At lower OBPF bandwidths the signal suffers from distortion and inter-symbol interference (ISI), while if the bandwidth is increased, no more phase-to-intensity conversion occurs in the case of OBPF demodulation. Even though a single OBPF with optimized bandwidth could be used for demodulation, only some categories of filters, such as Bragg gratings, are suitable for balanced detection [10]. MRR demodulation enables the simultaneous generation of two complementary signals at the drop and through ports, thus allowing balanced detection, which will be detailed further in Section II-D.

\section{Wavelength Detuning Sensitivity}

In real applications, some wavelength detuning may exist between the centre wavelength of the NRZ-DPSK signal and the transfer function of the MRR. Fig. 4(a) illustrates the simulated wavelength detuning sensitivity of the MRR and MZDI demodulations without OBPF. One can find that for MZDI demodulation, the OSNR requirements for the destructive (AMI) and constructive (DB) signals exhibit similar wavelength detuning dependence. For MRR demodulation, both through (AMI) and drop (DB) signals reveal better wavelength detuning sensitivity. The best wavelength detuning sensitivity can be obtained for the DB signal, but the OSNR requirement is about $6 \mathrm{~dB}$ higher than for the other schemes. On the other hand, the bandwidth of the de-multiplexing OBPF also plays an important role in the wavelength detuning tolerance for both MRR and MZDI demodulation. Fig. 4(b) illustrates the wavelength detuning tolerance (specified at $3 \mathrm{~dB}$ OSNR requirement degradation) as a (a)

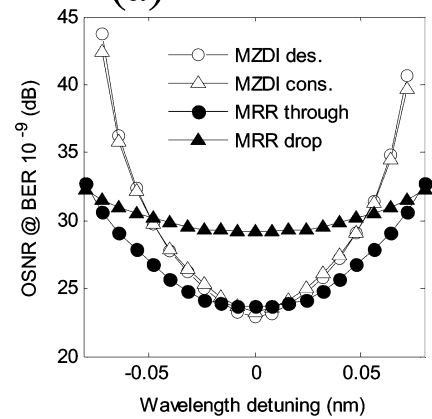

(b)

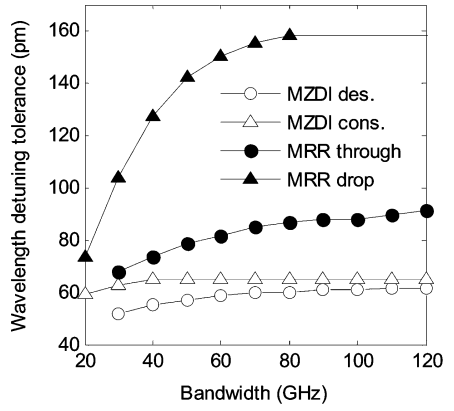

Fig. 4. (a) Simulated required OSNR (to obtain a BER of $10^{-9}$ ) versus wavelength detuning for the $40 \mathrm{Gbit} / \mathrm{s}$ AMI and DB signals demodulated using a MRR or an MZDI. (b) Wavelength detuning tolerance (at $3 \mathrm{~dB}$ OSNR requirement degradation) for the MZDI and MRR demodulation for different bandwidths of the de-multiplexing OBPF. $a=0.96$ and $|\kappa|^{2}=0.63$ are used for the MRR.

function of the OBPF bandwidth for the different demodulation schemes. One can find that the wavelength detuning tolerance of the MRR demodulation is better than that of the MZDI demodulation, especially for drop demodulation. However, as the bandwidth of the OBPF decreases, the wavelength detuning tolerance will be degraded more for MRR demodulation than for MZDI demodulation.

\section{Balanced Detection}

One of the well known benefits of DPSK modulation is its $\sim 3 \mathrm{~dB}$ sensitivity advantage compared to on-off keying (OOK) when balanced detection is used [1]. It is therefore important to check if the same advantage holds in the case of MRR demodulation. Furthermore, since the performance of balanced receivers used in conjunction with MZDI demodulators is known to be dependent on the optical bandwidth [17], [18], it is important to assess the optical bandwidth tolerance of balanced receivers employing MRRs. For this purpose, the OSNR sensitivity of balanced detection is calculated as a function of the optical bandwidth for demodulation using an MRR with $|\kappa|^{2}=$ 0.63 and compared to the case of MZDI demodulation. In order to ensure an appropriate model is used, the BER is calculated using error counting and the sensitivity is now defined as the OSNR required to obtain a BER of $3.3 \times 10^{-3}$ at the bit rate of $42.7 \mathrm{Gbit} / \mathrm{s}$ (corresponding to a BER of $10^{-12}$ when forward error correction with a 7\% redundancy ratio is used). Error counting is aborted after a minimum of 100 errors are detected. The results are shown in Fig. 5. Since the numerical approach is different from the one used to generate the results in the previous sections, it is important to ensure both methods are consistent. The OSNR requirement is also calculated using the Karhunen-Loève expansion method at $42.7 \mathrm{Gbit} / \mathrm{s}$ for a target BER of $3.3 \times 10^{-3}$ for single-ended detection at the through and drop ports of the MRR. The results are compared to those of error counting simulations in Fig. 5, showing good agreement. It can be seen that balanced detection used in conjunction with MRR demodulation presents a $\sim 3 \mathrm{~dB}$ sensitivity advantage compared to single-ended detection at the through port for a typical optical bandwidth value of $50 \mathrm{GHz}$, while it only performs $\sim 1.5 \mathrm{~dB}$ worse than when an MZDI is used. 


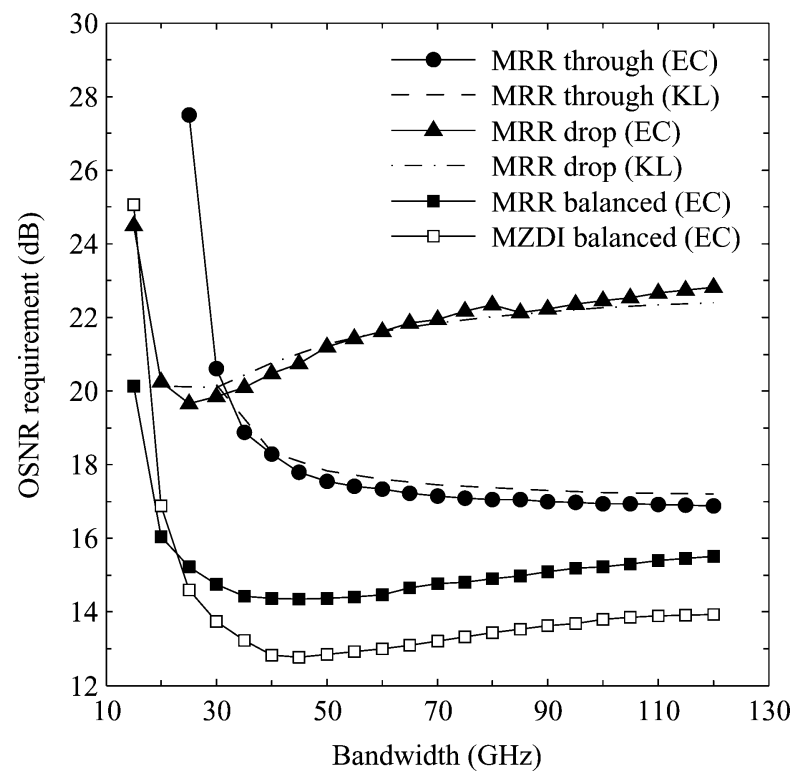

Fig. 5. OSNR requirement at a BER of $3.3 \times 10^{-3}$ for a $42.7 \mathrm{Gbit} / \mathrm{s} \mathrm{NRZ-}$ DPSK signal demodulated using an MRR with $|\kappa|^{2}=0.63$ and an MZDI. Single-ended detection at the through and drop ports, as well as balanced detection are considered for the MRR, while only balanced detection is considered for the MZDI. For MRR single-ended detection, sensitivities calculated using direct error counting (EC) and the Karhunen-Loève (KL) method are compared.
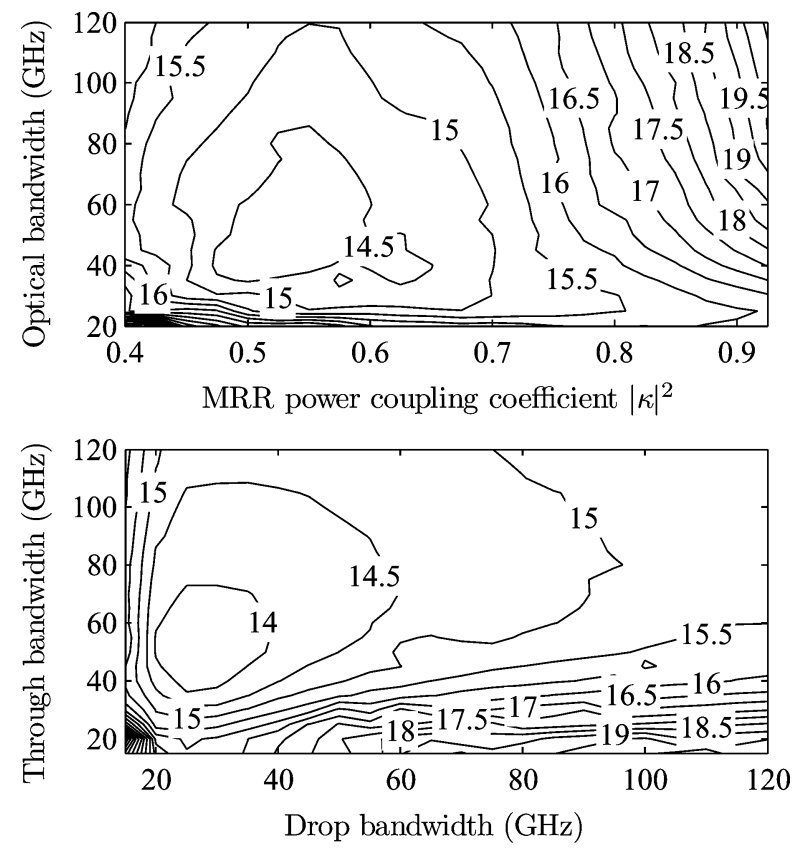

Fig. 6. Top: OSNR sensitivity at a BER of $3.3 \times 10^{-3}$ as a function of MRR power coupling ratio and optical bandwidth. (Bottom) OSNR sensitivity as a function of drop and through port bandwidths for an MRR power coupling ratio of $|\kappa|^{2} \sim 0.63$.

The joint optimization of the optical bandwidth and the MRR power coupling coefficient performed in Section B for single-ended detection is repeated for balanced detection. The results are shown in Fig. 6 where it can be seen that an optimum exists for a power coupling coefficient of $|\kappa|^{2} \sim 0.55$ and an optical bandwidth of $\sim 50 \mathrm{GHz}$, with a relatively large tolerance at this low required BER value. It should be pointed out however

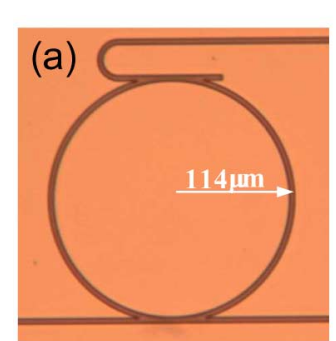

(c)

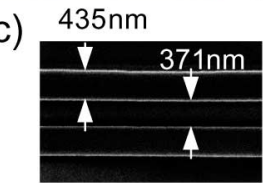

(e)

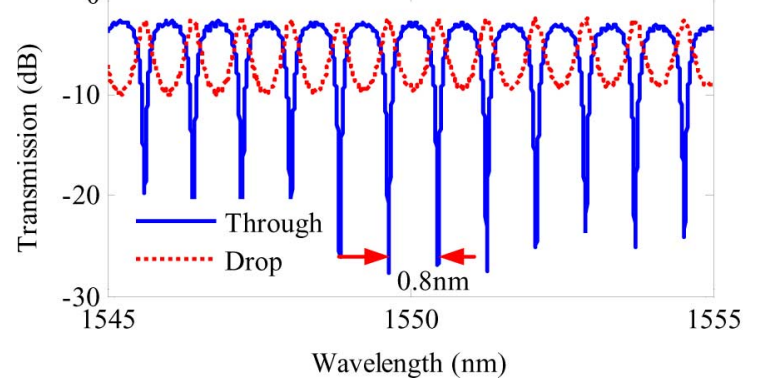

Fig. 7. (a) Optical microscope top view image of the fabricated device. (b) Design of the nano-taper coupler. (c), (d) Scanning electron microscope (SEM) pictures of the coupling region and the nano-taper. (e) Measured transmissions for the $\mathrm{TM}_{0}$ mode of the through and drop ports of the fabricated MRR.

that, in case balanced detection is used, the receiver structure is somehow different than in the single-ended detection study, with now a DPSK modulated signal at the receiver input (where the OSNR is defined), while it used to be an OOK signal (i.e., after phase-to-intensity modulation conversion in the MRR or MZDI) in the investigation of single-ended detection. Nevertheless, since it was shown in Section B that the optimum optical bandwidths are different for drop and through demodulation, one may wonder whether an independent adjustment of the drop and through ports bandwidths may affect the demodulation performance. Those two bandwidths are consequently varied independently, and the resulting OSNR sensitivity is also represented in Fig. 6. It appears that optimum performance is indeed achieved for different optical bandwidths at the through $(50 \mathrm{GHz})$ and drop $(25 \mathrm{GHz})$ ports. This however only results in a marginal improvement of the receiver sensitivity, of the order of $\sim 0.5-1 \mathrm{~dB}$ over a broad bandwidth range of 30-90 $\mathrm{GHz}$, compared to the situation when the optical bandwidth is identical at both ports. Consequently, MRR demodulation is also shown to be a promising solution when used in conjunction with balanced detection.

\section{EXPERIMENTAL DEMONSTRATION}

\section{A. Device Fabrication and Characterization}

The silicon MRR was fabricated on a silicon-on-insulator (SOI) wafer with top silicon thickness of $250 \mathrm{~nm}$ and buried silicon dioxide of $3 \mu \mathrm{m}$. First, diluted (1:1 in anisole) electron-beam resist ZEP520A was spin-coated on the wafer to form 


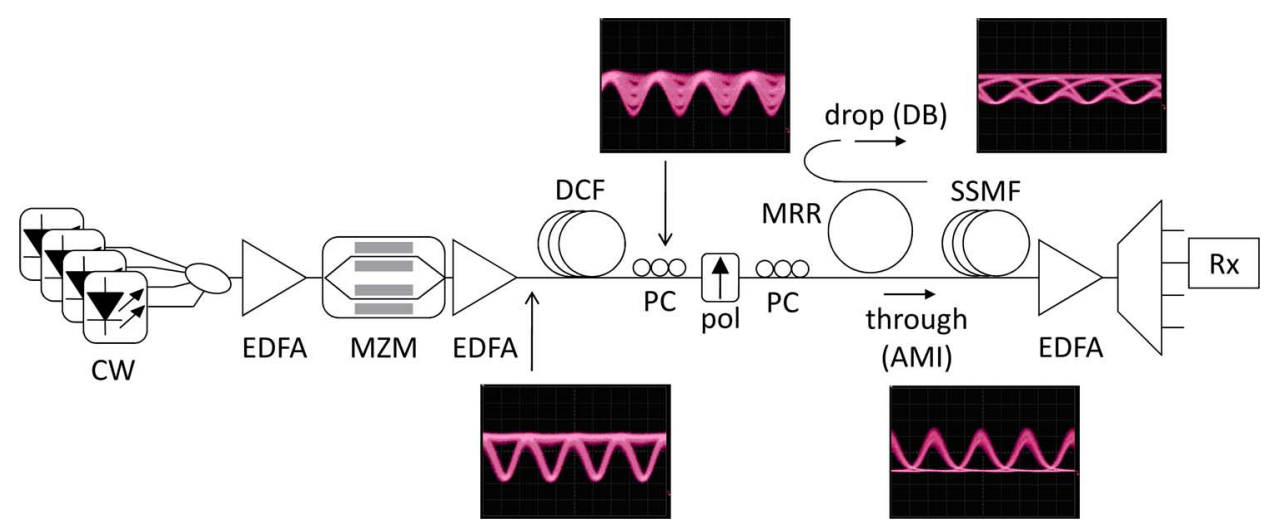

Fig. 8. Experimental setup for MRR based WDM NRZ-DPSK demodulation. The insets show the measured waveforms of the combined WDM signal at the output of the NRZ-DPSK modulator and after the de-correlation fiber, as well as the eye diagrams of a single channel at the drop and through ports of the MRR. For the filtering bandwidth dependence investigation, the AWG is replaced by an OBPF. For MZDI based demodulation the MRR is replaced by a 1-bit MZDI.

a $110 \mathrm{~nm}$-thick mask layer. The structure of the device was then defined using electron-beam lithography (JEOL JBX-9300FS). After that, the sample was etched by inductively coupled plasma reactive ion etching (ICP-RIE) to transfer the patterns to the top silicon layer. A layer of $3.5 \mu \mathrm{m}$ thick polymer (SU8-2005) was spin-coated afterwards as a top cladding layer. Top cladding regions and nano-couplers are defined by electron-beam lithography, and finally formed by developing. The radius of the MRR is $114 \mu \mathrm{m}$, as shown in Fig. 7(a), with a waveguide width of 435 $\mathrm{nm}$ and coupling gap of $371 \mathrm{~nm}$ for both through and drop coupling regions, as shown in Fig. 7(c). The silicon waveguide is inversely tapered to $48 \mathrm{~nm}$, covered by a polymer waveguide (see Figs. 7(b) and (d)) to form a nano-coupler, resulting in ultra-low coupling loss [19] to the fiber. Fig. 7(e) shows the measured transmissions of the through and drop ports of the MRR for the $\mathrm{TM}_{0}$ mode as a function of wavelength. The extinction ratio (ER) of the through transmission is as high as $25 \mathrm{~dB}$, the measured FSR is $0.8 \mathrm{~nm}$, corresponding to $100 \mathrm{GHz}$, and the Q value is 6700 . The total insertion loss of the device is only $3 \mathrm{~dB}$. Similarly to normal silicon nano-waveguides, the transmission of the device is polarization dependent. To reduce the polarization dependence, a polarization diversity structure can be introduced [20], [21].

\section{B. Experimental Set-Up}

The experimental setup for WDM NRZ-DPSK demodulation is illustrated in Fig. 8. Four channels of continuous wave (CW) laser light with wavelengths of $1548.65 \mathrm{~nm}, 1550.26 \mathrm{~nm}$, $1551.90 \mathrm{~nm}$, and $1553.54 \mathrm{~nm}$ (channel spacing of $\sim 200 \mathrm{GHz}$ ) are combined in a coupler, amplified by an erbium-doped fiber amplifier (EDFA), then simultaneously modulated in the NRZDPSK format in a Mach-Zehnder modulator. The bit rate is 40 Gbit/s and the bit pattern length is $2^{31}-1$. The WDM NRZDPSK channels are then amplified by another EDFA and de-correlated in $150 \mathrm{~m}$ dispersion-compensating fiber (DCF) with total dispersion of $-17.4 \mathrm{ps} / \mathrm{nm}$ at $1550 \mathrm{~nm}$. In order to ensure that all WDM channels are optimally coupled to the MRR, a polarization controller (PC) followed by a polarizer is first used to align all channels to the same polarization state. A second $\mathrm{PC}$ is then used to align the input polarization to the TM mode of the waveguide. Demodulated AMI and DB signals will be obtained at the through and drop ports of the MRR, respectively. Afterwards, $1 \mathrm{~km}$ standard single mode fiber (SMF) is used to compensate the dispersion introduced by the DCF. The WDM demodulated signal is amplified by another EDFA, de-multiplexed by an OBPF or an AWG and finally detected in an optically preamplified receiver. In the experiments, the receiver power sensitivity at a BER of $10^{-9}$ is adopted as a performance metric.

\section{Filtering Bandwidth Dependence}

The influence of the bandwidth of the OBPF is experimentally analyzed for single channel operation, in which only channel 2 is switched on, as shown in Fig. 9. Fig. 9(a) shows the measured BERs of the demodulated AMI and DB signals at the through and drop ports of the MRR for different bandwidths of the OBPF. Reference curves where no OBPF is used are also shown. Fig. 9(b) represents the corresponding receiver sensitivities at a BER of $10^{-9}$. One can find that, as the bandwidth of the OBPF decreases, the receiver sensitivity of the AMI signal demodulated at the through port degrades. However, an improved receiver sensitivity of the DB signal demodulated at the drop port is obtained as the bandwidth decreases over the investigated range. This is in qualitative agreement with the simulation results of Fig. 3. The measured curves however quantitatively differ from the simulated ones due to the presence of OBPFs with different transfer functions in the experimental implementation. Furthermore, the performance is also affected by the frequency response of the OBPFs employed in order to suppress out-of-band ASE noise in the preamplified receiver.

\section{Wavelength Detuning Tolerance}

Wavelength detuning tolerances for both through and drop port demodulations are also experimentally characterized, as illustrated in Fig. 10. The receiver sensitivity is evaluated at a BER of $10^{-9}$ for single channel operation without AWG. For the demodulation with a commercial fiber MZDI having a $42.7 \mathrm{GHz}$ FSR, the receiver sensitivities for both constructive and destructive ports exhibit similar dependences to the wavelength detuning, with allowed detuning ranges for $3 \mathrm{~dB}$ power penalty of about 0.04 and $0.05 \mathrm{~nm}$ for the demodulated AMI and DB signals, respectively. A similar wavelength detuning sensitivity is obtained for MRR through port demodulation, with a detuning 

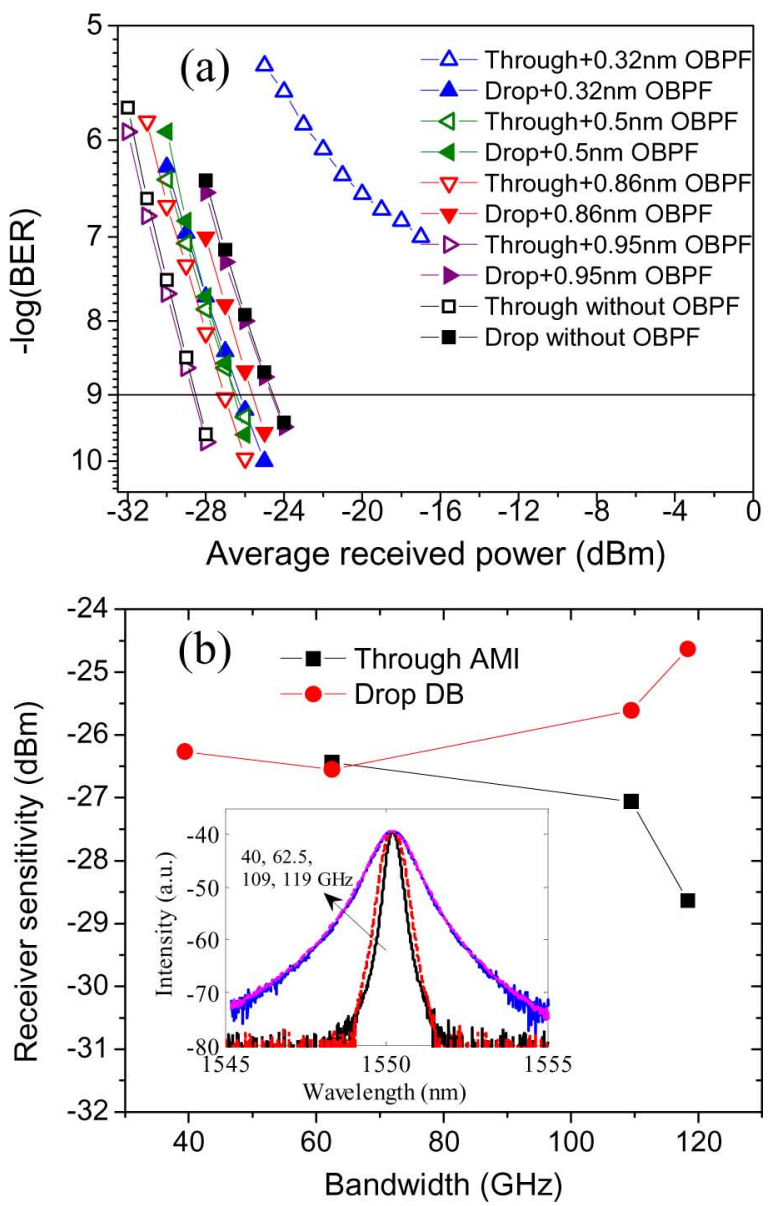

Fig. 9. (a) BER measurements of the demodulated AMI and DB signals for different bandwidths of the OBPF, as well as in the reference case when no OBPF is used. (b) corresponding power penalties at a BER of $10^{-9}$ compared to the case without OBPF.

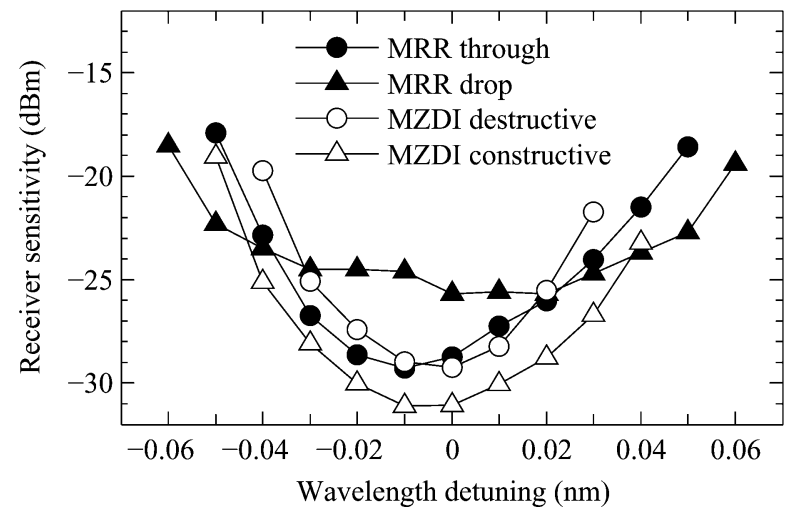

Fig. 10. Measured receiver sensitivity versus wavelength detuning for the AMI and DB signals demodulated by MRR or MZDI.

tolerance of about $0.05 \mathrm{~nm}$. The best wavelength detuning sensitivity is obtained for drop port demodulation, with a wavelength detuning tolerance of $0.09 \mathrm{~nm}$ (all at $3 \mathrm{~dB}$ receiver sensitivity degradation). Those results are also in qualitative agreement with the simulations. The narrower wavelength detuning tolerance compared to the one predicted in the simulations is attributed to the impact of the ASE suppressing OBPFs in the preamplified receiver.

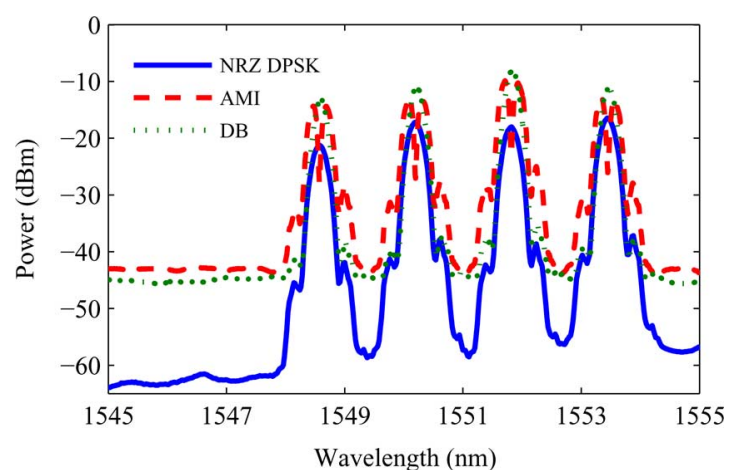

Fig. 11. Measured spectra of the WDM NRZ-DPSK signals, as well as of the WDM AMI and DB signals demodulated in a single MRR.
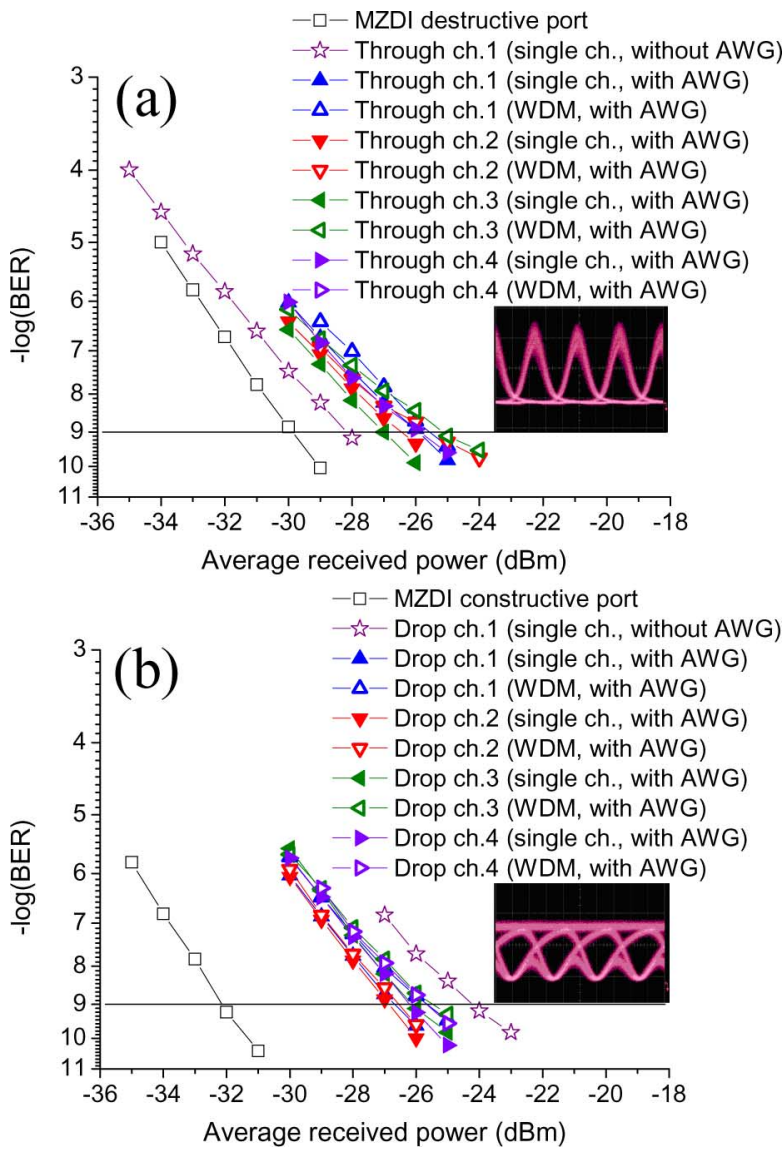

Fig. 12. BER measurements for the multiple channel (a) AMI and (b) DB signals demodulated by the MRR, as well as a single channel AMI and DB signal demodulated by the MZDI. The insets show typical eye diagrams for the AMI and DB signals demodulated by the MRR.

\section{E. WDM Operation}

Fig. 11 shows the measured spectra of the WDM NRZ-DPSK signals, as well as of the WDM AMI and DB signals demodulated in a single MRR. The notches of the through transfer function and the peaks of the drop transfer function of the MRR are aligned to the carrier wavelengths of all the WDM channels, resulting in simultaneous demodulation of all channels to the AMI and DB formats, respectively. Fig. 12(a) and (b) presents BER measurements for all channels at the output of the through (AMI) and drop (DB) ports. The inset pictures illustrate very 
clear eye-diagrams for the demodulated AMI and DB signals. For comparison purpose, the MRR is also substituted with a commercial fiber MZDI with $42.7 \mathrm{GHz}$ FSR used for single channel demodulation. Although the BER for both through and drop port demodulations are worse than that of the MZDI, all the four channels reach bit error rates below $10^{-9}$, without noticeable error floor. For through port demodulation, the AWG induces about $1-2 \mathrm{~dB}$ power penalty, and about $0.5-1 \mathrm{~dB}$ power penalty is further caused by the crosstalk between channels. However, for drop port demodulation, the AWG improves the receiver sensitivity by about $3 \mathrm{~dB}$, and around $0.5 \mathrm{~dB}$ power penalty is further introduced by the crosstalk. As there is a trade off between the performance of the demodulated AMI and DB signals [13], it is found that the MRR with $Q$ value of 6700 associated with the AWG, lead to similar receiver sensitivities for both through and drop port demodulations.

\section{CONCLUSION}

We have comprehensively analyzed the performance of WDM NRZ-DPSK demodulation at $40 \mathrm{Gbit} / \mathrm{s}$ based on a single MRR. A detailed comparison between the MRR, MZI and OBPF demodulations has been made, which shows the advantages of MRR based WDM NRZ-DPSK demodulation. The scheme has also been shown to be promising in case balanced detection is used. Multiple channels WDM NRZ-DPSK demodulation at $40 \mathrm{Gbit} / \mathrm{s}$ has been demonstrated by a single silicon MRR with FSR of $100 \mathrm{GHz}$ and Q value of 6700 . Both the through and drop port demodulations exhibit very good and similar BER performance for all the channels. Better wavelength detuning sensitivity is obtained for drop port demodulation compared to the MZDI demodulation.

\section{ACKNOWLEDGMENT}

One of the authors, Y. Ding, would like to thank the Chinese Scholar Council (CSC) for the support and K. Yvind and D. Larsson for the supply of the probe station.

\section{REFERENCES}

[1] A. H. Gnauck and P. J. Winzer, "Optical phase-shift-keyed transmission," J. Lightw. Technol., vol. 23, no. 1, pp. 115-130, Jan. 2005.

[2] I. P. Kaminow, "Balanced optical discriminator," Appl. Opt., vol. 3, no. 4, pp. 507-510, 1964.

[3] X. Liu, A. H. Gnauck, X. Wei, J. Y. C. Hsieh, and V. Chien, "Athermal optical demodulator for OC-768 DPSK and RZ-DPSK signals," IEEE Photon. Technol. Lett., vol. 17, no. 12, pp. 2610-2612, Dec. 2005.

[4] E. Ciaramella, G. Contestabile, and A. D'Errico, "A novel scheme to detect optical DPSK signals," IEEE Photon. Technol. Lett., vol. 16, no. 9, pp. 2138-2140, Sept. 2004.

[5] C. W. Chow and H. K. Tsang, "Polarization-independent DPSK demodulation using a birefringent fiber loop," IEEE Photon. Technol. Lett., vol. 17, no. 6, pp. 1313-1315, June 2005.

[6] I. Lyubomirsky and C.-C. Chien, "DPSK demodulator based on optical discriminator filter," IEEE Photon. Technol. Lett., vol. 17, no. 2, pp. 492-494, Feb. 2005.

[7] M. P. Fok and C. Shu, "Delay-asymmetric nonlinear loop mirror for DPSK demodulation," Opt. Lett., vol. 33, no. 23, pp. 2845-2847, Dec. 2008.

[8] C. R. Doerr, M. A. Cappuzzo, E. Y. Chen, A. Wong-Foy, L. T. Gomez, S. S. Patel, S. Chandrasekhar, and A. E. White, "Polarization-insensitive planar lightwave circuit dual-rate Mach-Zehnder delay-interferometer," IEEE Photon. Technol. Lett., vol. 18, no. 16, pp. 1708-1710, Aug. 2006.
[9] A. D'Errico, R. Proietti, L. Giorgi, G. Contestabile, and E. Ciaramella, "WDM-DPSK detection by means of frequency-periodic Gaussian filtering," Electron. Lett., vol. 42, no. 2, pp. 112-114, Jan. 2006.

[10] L. Christen, S. Nuccio, Y. K. Lize, A. E. Willner, and L. Paraschis, "Simultaneous balanced DPSK demodulation of multiple $40 \mathrm{Gbit} / \mathrm{s}$ WDM channels using a single periodic FBG," in Lasers Electro-Opt./ Quantum Electron. Laser Sci. Conf. Photon. Applic. Syst. Technol. OSA Tech. Dig., 2007, paper CMJJ3.

[11] D. O. Caplan, M. L. Stevens, and J. J. Carney, "High-sensitivity multi-channel single-interferometer DPSK receiver," Opt. Exp. vol. 14, no. 23, pp. 10 984-10 989, Nov. 2006 [Online]. Available: http://www.opticsinfobase.org/abstract.cfm?URI=oe-14-23-10984, [Online]. Available:

[12] Y. Yu, J. B. RosasFernández, X. L. Zhang, D. X. Huang, R. V. Penty, and I. H. White, "Novel and flexible WDM NRZ-DPSK system with demultiplexing and demodulation using a single standard AWG," in Opt. Fiber Commun. Conf. Tech. Dig., 2009, paper OThF7.

[13] L. Zhang, J.-Y. Yang, M. Song, Y. Li, B. Zhang, R. G. Beausoleil, and A. E. Willner, "Microring-based modulation and demodulation of DPSK signal," Opt. Express vol. 15, no. 18, pp. 11 564-11 569, Sept. 2007 [Online]. Available: http://www.opticsinfobase.org/abstract.cfm?URI=oe-15-18-11564, [Online]. Available:

[14] L. Xu, C. Li, C. Wong, and H. K. Tsang, "Optical differential-phaseshift-keying demodulation using a silicon microring resonator," IEEE Photon. Technol. Lett., vol. 21, no. 5, pp. 295-297, Mar. 2009.

[15] P. Serena, Optilux-An Open Source Optical System Simulator [Online]. Available: http://optilux.sourceforge.net Available:

[16] E. Forestieri, "Evaluating the error probability in lightwave systems with chromatic dispersion, arbitrary pulse shape and pre- and postdetection filtering," J. Lightw. Technol., vol. 18, no. 11, pp. 1493-1503, Nov. 2000

[17] P. J. Winzer, S. Chandrasekhar, and H. Kim, "Impact of filtering on RZ-DPSK reception," IEEE Photon. Technol. Lett., vol. 15, no. 6, pp. 840-842, Jun. 2003.

[18] Y. K. Lizé, X. Wu, M. Nazarathy, Y. Atzmon, L. Christen, S. Nuccio, M. Faucher, N. Godbout, and A. E. Willner, "Chromatic dispersion tolerance in optimized NRZ-, RZ- and CSRZ-DPSK demodulation," Opt. Exp. vol. 16, no. 6, pp. 4228-4236, Mar. 2008 [Online]. Available: http://www.opticsinfobase.org/abstract.cfm?URI=oe-16-6-4228, [Online]. Available:

[19] M. Pu, L. H. Frandsen, H. Ou, K. Yvind, and J. M. Hvam, "Low insertion loss soi microring resonator integrated with nano-taper couplers," in Frontiers in Optics Tech. Dig., 2009, paper FThE1.

[20] T. Barwicz, M. R. Watts, A. Popovic Milos, P. T. Rakich, L. Socci, F. X. Kartner, E. P. Ippen, and H. I. Smith, "Polarization-transparent microphotonic devices in the strong confinement limit," Nat. Photon., vol. 1, no. 1, pp. 57-60, Jan. 2007.

[21] H. Fukuda, K. Yamada, T. Tsuchizawa, T. Watanabe, H. Shinojima, and S.-I. Itabashi, "Silicon photonic circuit with polarization diversity," Opt. Exp., vol. 16, no. 7, pp. 4872-4880, Mar. 2008.

Yunhong Ding received the B.S. degree in photonic information science and technology (with first-class honors) from Huazhong University of Science and Technology (HUST), Wuhan, China, in 2006. He is currently working toward the Ph.D. degree in electronic science and technology at Division of Opto-Electronic Devices \& Integration (OEDI), Wuhan National Laboratory for Optoelectronics (WNLO); College of Optoelectronic Science and Engineering, Huazhong University of Science and Technology, Wuhan, China.

$\mathrm{He}$ is currently working as a guest Ph.D. student with the Department of Photonics Engineering, Technical University of Denmark (DTU), Lyngby, Denmark. His research interests are optoelectronic devices and optoelectronic integration, and numerical modeling, design and fabrication of semiconductor microring and microresonator devices.

Jing Xu received the Ph.D. degree from Wuhan National Laboratory for Optoelectronics, Huazhong University of Science and Technology, Wuhan, China, in 2009.

She is currently a Postdoctoral Researcher with DTU Fotonik, Department of Photonics Engineering, Technical University of Denmark, Lyngby, Denmark. Her current research interests include high-speed optical signal processing and high-speed optical time-division multiplexing systems. 
Christophe Peucheret received the engineering degree from Ecole Nationale Supérieure des Télécommunications de Bretagne, Brest, France, in 1994, the M.Sc. degree in microwaves and optoelectronics from University College London, London, U.K., in 1994, and the Ph.D. degree from the Technical University of Denmark (DTU), Copenhagen, Denmark, in 2003.

He is currently an Associate Professor with the Department of Photonics Engineering at DTU, working in the field of optical modulation formats and all-optical signal processing.

Minhao Pu received the B.S. degree in optoelectronic information engineering from Tianjin University, Tianjin, China, in 2005, and the M.S. degree in photonics from the Royal Institute of Technology (KTH), Kista, Sweden, in 2007. $\mathrm{He}$ is currently working toward the Ph.D. degree at the Department of Photonics Engineering, DTU Fotonik, Technical University of Denmark, Lyngby, Denmark.

His research interests include design, fabrication and application of silicon photonic devices including photonic crystal and microring resonators.

Liu Liu received the B.Eng. degree in information engineering from Zhejiang University, Hangzhou, China, in 2002, and the Ph.D. degree in photonics from Royal Institute of Technology, Stockholm, Sweden, in 2006.

From 2007 to 2009, he was a Postdoctoral Researcher with Photonics Research Group, Department of Information Technology, Ghent University, Ghent, Belgium. He is currently in the Department of Photonics Engineering, Technical University of Denmark, Lyngby, Denmark. His research interests include heterogeneous integration and silicon nanophotonic devices.

Jorge Seoane received the Telecommunication Engineer degree from the Faculty of Engineering, Bilbao, Spain, and the Ph.D. degree in optical communication from DTU Fotonik, Technical University of Denmark, Lyngby, Denmark.

His work in the optical field includes contributions in migration scenarios from 10 to $40 \mathrm{Gbit} / \mathrm{s}$ in WDM systems, 100-G Ethernet systems, optical label switching and advanced modulation formats applied to both WDM and OTDM systems. His current research interests include all-optical signal processing, advanced modulation formats, and novel methods for their amplification.
Haiyan Ou received the M.Sc. degree in semiconductor devices and microelectronics with minor in industrial enterprise management in Department of Solid State Electronics, Huazhong University of Science and Technology (HUST), Wuhan, Hubei, China, in 1997, and the Ph.D. degree in optical communication in the Institute of Semiconductors, Chinese Academy of Sciences (CAS), Beijing, China, in 2000.

She has been with the Department of Photonics Engineering, Technical University of Denmark (DTU Fotonik), Lyngby, Denmark, since 2000, first as an Assistant Professor and currently an Associate Professor. Her research interests are LEDs, fiber optics, integrated optics, nano-materials, and energy-efficient devices.

Xinliang Zhang received the Ph.D. degree from Huazhong University of Science and Technology (HUST), Wuhan, China, in 2001.

$\mathrm{He}$ is currently a Professor with Wuhan National Laboratory for Optoelectronics and Institute of Optoelectronics Science and Engineering, Huazhong University of Science and Technology, Wuhan, Hubei, China.

His current research interests are all-optical signal processing and related components. He has authored or coauthored 50 related papers in international journals or conferences proceedings.

Dexiu Huang graduated from the Department of Radio Engineering, Huazhong Institute of Technology (now Huazhong University of Science and Technology), Wuhan, China, in 1963.

Since then, he has been with the same university as Assistant Professor, Lecturer, Associate Professor, and Professor in 1963, 1978, 1986, and 1990, respectively. Prior to 1972, he was engaged in research on semiconductor devices and passive devices in radio engineering. From 1972 to 1981, he performed research on solid-state lasers and applications. From 1981 to 1983, he was a Visiting Scientist with the Oregon Graduate Center, focusing on semiconductor optoelectronic devices. Since then, he has been in the field of optical communication performing research on semiconductor optoelectronics devices and some passive devices. He is currently a Professor of the College of Information Science and Engineering. He is the author of five books and has authored or coauthored more than 200 papers. 